\title{
Yule-Nielsen effect in halftone prints: Graphical analysis method and improvement of the Yule-Nielsen transform
}

\author{
Mathieu Hébert ${ }^{\mathrm{a}, \mathrm{b}}$ \\ ${ }^{a}$ Université de Lyon, Université Jean Monnet de Saint-Etienne, CNRS UMR5516 Laboratoire \\ Hubert Curien, 18 rue Benoît Lauras, F-42000 Saint-Etienne, France. ' Institut d'Optique, 18 rue \\ Benoît Lauras, F-42000 Saint-Etienne, France.
}

\begin{abstract}
The well-known Yule-Nielsen modified spectral Neugebauer model is one of the most accurate predictive models for the spectral reflectance of printed halftone colors which expresses the spectral reflectance of halftones raised to the power $1 / n$ as a linear combination of the spectral reflectance of the fulltone colors (Neugebauer primaries) also raised to the power $1 / n$, where $n$ is a tunable real number. The power $1 / n$ transform, characteristic of the Yule-Nielsen transform, empirically models the nonlinear relationship between the spectral reflectances of halftones and fulltones due to the internal propagation of light by scattering into the printing support, a phenomenon known as "optical dot gain" or "YuleNielsen effect". In this paper, we propose a graphical method permitting to observe this non-linear relationship in the case of single-ink halftones and to experimentally check the capacity of the Yule-Nielsen model to predict it accurately. In the case where the Yule-Nielsen transform is not well adapted to the considered type of prints, we propose alternative transforms in order to improve the prediction accuracy.
\end{abstract}

Keywords: Color reproduction, spectral model, halftone color, printing, reflectance, transmittance, Yule-Nielsen model.

\section{INTRODUCTION}

Digital printing technologies have considerably increased the number of applications and printable materials in a context where designers and even customers themselves are more and more used to select the printing materials and parameters in order to precisely obtain the expected appearance. Precise color management needs a calibration of the printing system for the selected printing support, inks and halftoning technique [1]. The classical calibration method is based on a color profile established by measuring the color of about one thousand of halftones [2], a procedure that must be repeated each time the printing support, the ink set or the halftoning technique is modified. The use of a color prediction model can considerably ease the color calibration since only a few tens of colors need to be printed and measured. One of the most accurate models available today is the Yule-Nielsen modified spectral Neugebauer model [3], or simply "spectral YuleNielsen model", which is widely used for the calibration of printing systems $[4,5,6,7]$ and can be improved by the method by Crété \& al. for estimating the effective surface coverage of the inks [8].

The spectral Yule-Nielsen model predicts the spectral reflectance of halftone raised to the power $1 / n$ as a linear combination of the spectral reflectances of the fulltone colors (i.e. colors made by covering the whole surface with one or several inks, also called "colorants" or "Neugebauer primaries") also raised to the power $1 / n$, where $n$ is a tunable parameter. The weights attributed to the different fulltone colors are the effective surface coverages of these colors in the halftone. The power $1 / n$ transform empirically models the nonlinear relationship between the spectral reflectances of halftones and fulltones due to the internal propagation of light by scattering into the printing support, a well-known phenomenon also called "optical dot gain" or "Yule-Nielsen effect" [9]. In this paper, we propose a graphical method showing this non-linear relationship in an intelligible way for single-ink halftones. The graph represents the reflectance of a single-ink halftone as a function of the reflectance of this fulltoned ink. If the spectral measurement contains $k$ wavebands, then $k$ points can be displayed in the graph and it is easy to check how well the Yule-Nielsen function matches them. The measured points generally follow a curved line which fairly coincides with the plot of the YuleNielsen transform computed with the appropriate $n$ value and effective ink surface coverage. In some cases however, the coincidence is not perfect and alternative transforms, based on other functions than the power $1 / n$ function, may be found. We propose a few ones which provide modest but appreciable gain in prediction accuracy, this latter being measured in terms of average CIELAB $\triangle \mathrm{E} 1994$ values over several tens of printed colors. The graphical method is also very interesting to display effects that are rather difficult to detect by looking directly at the spectral reflectances. 
SPIE Electronic Imaging - Color Imaging XIX: Displaying, Processing, Hardcopy, and Applications, 2-6 February 2014, 9015-27.

\section{SPECTRAL REFLECTANCE AND TRANSMITTANCE OF HALFTONES COLORS}

Before analyzing the relevance of the spectral Yule-Nielsen model for a given type of print, we propose to recall in this section the whole prediction method, including the ink spreading assessment proposed by Hersch and Crété [8]. The model is presented for reflectance of CMY halftones but it similarly applies for transmittance and for any ink colors.

\subsection{Halftone colors}

Halftoning is a color reproduction technique based on a finite set of coloring substances, the inks, which are deposited according to binary grids of small patterns (inks dots). The halftone surface is a mosaic of colored areas corresponding to the partial overlap of the ink dots. The areas with no ink, those with a single ink layer, and those with two or three superposed ink layers are each one considered as distinct colorants (also called Neugebauer primaries). In CMY printing, based on cyan, magenta and yellow inks, one obtains eight colorants corresponding to the areas with no ink, cyan alone, magenta alone, yellow alone, red (magenta and yellow), green (cyan and yellow), blue (cyan and magenta) and black (cyan, magenta and yellow).

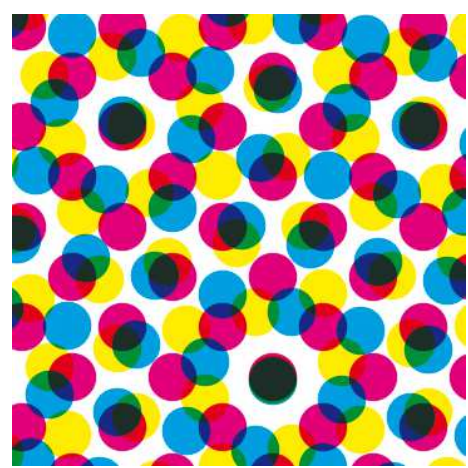

Figure 1. Detail of a halftone color with cyan, magenta and yellow inks, showing the ink dots and the mosaic of 8 colorants.

In classical clustered-dot or error diffusion prints, the fractional area occupied by each colorant can be deduced from the surface coverages of the primary inks according to Demichel's equations [12]. Denoting as $c, m$, and $y$ the surface coverages of the cyan, magenta and yellow inks, the surface coverages of the eight colorants are:

$$
\begin{aligned}
a_{w} & =(1-c)(1-m)(1-y) & a_{m+y} & =(1-c) m y \\
a_{c} & =c(1-m)(1-y) & a_{c+y} & =c(1-m) y \\
a_{m} & =(1-c) m(1-y) & a_{c+m} & =c m(1-y) \\
a_{y} & =(1-c)(1-m) y & a_{c+m+y} & =c m y
\end{aligned}
$$

\subsection{The Yule-Nielsen model}

The first color prediction model for halftone prints, proposed by Neugebauer [13], assumes that the color of the halftone is a linear combination of the colors of the colorants contained in it. The spectral version of this model can be written:

$$
R(\lambda)=\sum_{i=1}^{8} a_{i} R_{i}(\lambda)
$$

where $R(\lambda)$ denotes the spectral reflectance of the printed halftone, $R_{i}(\lambda)$ the spectral reflectances of the colorants $i$ printed each one on a large area to be measured, and $a_{i}$ the respective surface coverages of the colorants in the halftone.

However, this linear equation (2) does not predict correctly the color of the halftone due to the scattering of light within the paper bulk which induces a color halo around the ink dots. This phenomenon, known as the "Yule-Nielsen effect", is even amplified by the multiple reflections between the paper bulk and the print-air interface [1]. In order to account for this effect, Vigiano [3] proposed to apply the Yule-Nielsen transform [9] on the spectral Neugebauer equation:

$$
R(\lambda)=\left[\sum_{i=1}^{8} a_{i} R_{i}^{1 / n}(\lambda)\right]^{n}
$$


SPIE Electronic Imaging - Color Imaging XIX: Displaying, Processing, Hardcopy, and Applications, 2-6 February 2014, 9015-27.

where $n$ is real number, generally higher than 1 (when $n=1$, the spectral Yule-Nielsen equation (3) is equivalent to the Neugebauer equation), to be fitted. It is generally related to the strength of scattering in the printed support [10], on the halftone screen frequency and on the ink-paper interaction [11].

\subsection{Ink spreading assessment}

At printing time, the inks may spread on the surface in different amounts according to the presence or not of other inks on the surface [9]. As a consequence, the effective surface coverages of the inks, thereby those of the colorants in the halftone, are not known. A solution to obtain them is to establish experimentally the correspondence between nominal and effective surface coverages through so-called ink spreading functions. Let us present the method to compute them.

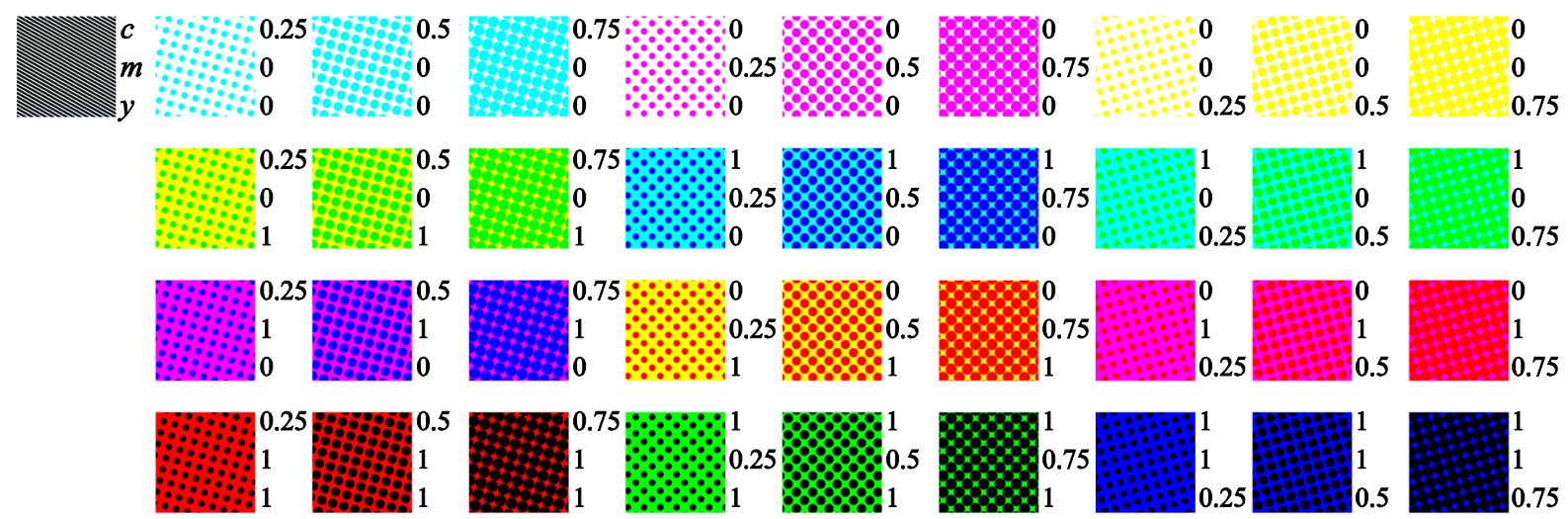

Figure 2. Halftones used for the ink spreading assessment.

The set of 36 halftones shown in Figure 2 are printed. They correspond to the combinations of one ink $u$ at nominal surface coverage $a=0.25,0.5$ or 0.75 , and one solid colorant $v$ obtained by printing each other ink at a surface coverage 0 or 1 . Each of these halftones contains two colorants corresponding to the areas where ink $u$ is superposed to colorant $v$ [effective surface coverage $a_{u / v}^{\prime}$, spectral reflectance $R_{u \& v}(\lambda)$ ] and to the areas where colorant $v$ is alone [effective surface coverage $1-a_{u / v}^{\prime}$, spectral reflectance $\left.R_{v}(\lambda)\right]$. According to the Yule-Nielsen equation (3), their reflectance $R(\lambda)$ is written:

$$
R(\lambda)=\left[\left(1-a_{u / v}^{\prime}\right) R_{v}^{1 / n}(\lambda)+a_{u / v}^{\prime} R_{u \& v}^{1 / n}(\lambda)\right]^{n}
$$

The effective surface coverage $a_{u / v}^{\prime}$ is fitted by minimizing the sum of square differences between the measured spectral reflectance of the halftone, $M(\lambda)$, and the spectral reflectance predicted by Eq. (4):

$$
a_{u / v}^{\prime}=\underset{0 \leq x \leq 1}{\arg \min \lambda} \sum_{\lambda=380 \mathrm{~nm}}^{730 \mathrm{~nm}} 1\left\{M()-\left[\lambda(-x) R_{v}^{1 / n}(\lambda)+x R_{u \& v}^{1 / n}()\right]^{n}\right\}^{2}
$$

Repeating this procedure for the 36 halftones yields 12 lists of 3 effective surface coverages $a_{u / v}^{\prime}$ associated with the nominal surface coverages $\{0.25,0.5,0.75\}$. It is assumed that the effective surface coverage is 0 , respectively 1 , when the nominal surface coverage is 0 (no ink), respectively 1 (full coverage). By linear interpolation, one obtains continuous curves $a^{\prime}=f_{u / v}(a)$, where $a$ and $a^{\prime}$ denote the nominal, respectively the effective surface coverages of the halftoned ink (see examples in Figure 3).

With the fitted surface coverages, the spectral reflectances predicted by Eq. (4) are as close as possible to the measured spectral reflectances but not equal to them. In order to assess the difference between predicted and measured spectra, we use a visual metric, the CIELAB $\Delta \mathrm{E}_{94}$ color distance, calculated by converting the two spectra first into CIE1931-XYZ tristimulus values by selecting the D65 illuminant, and then into CIELAB color coordinates using as white reference the spectral reflectance of the unprinted paper illuminated with the D65 illuminant [14].

The optimal $n$ value can be determined by trying successively different values, e.g. $n=1$ to 10 in steps of 0.1 , and keep the $n$ value for which the average $\Delta \mathrm{E}_{94}$ value computed over the 36 halftones is minimal. Figure 4 shows two plots of the average $\Delta \mathrm{E}_{94}$ value as a function of the $n$ value for CMY colors printed in inkjet with the Canon PixmaPro 9500 Mark II 
SPIE Electronic Imaging - Color Imaging XIX: Displaying, Processing, Hardcopy, and Applications, 2-6 February 2014, 9015-27.

printer (a) at 150 lpi on Canon PP201 $260 \mathrm{~g} / \mathrm{m}^{2}$ glossy coated paper (spectral measurements based on the $45^{\circ}: 0^{\circ}$ geometry using the X-rite i1 spectrophotometer) and (b) at 100 lpi on common $80 \mathrm{~g} / \mathrm{m}^{2}$ office paper (spectral measurements based on the diffuse: $8^{\circ}$ geometry using the X-rite Color i7 spectrophotometer). A minimum is clearly displayed in the case of the coated paper at $n=3.5$, whereas the curve asymptotically decreases in the case of the office paper, which indicated that the optimal $n$ tends to infinity (in practice, we can set $n$ to 20 or 100)
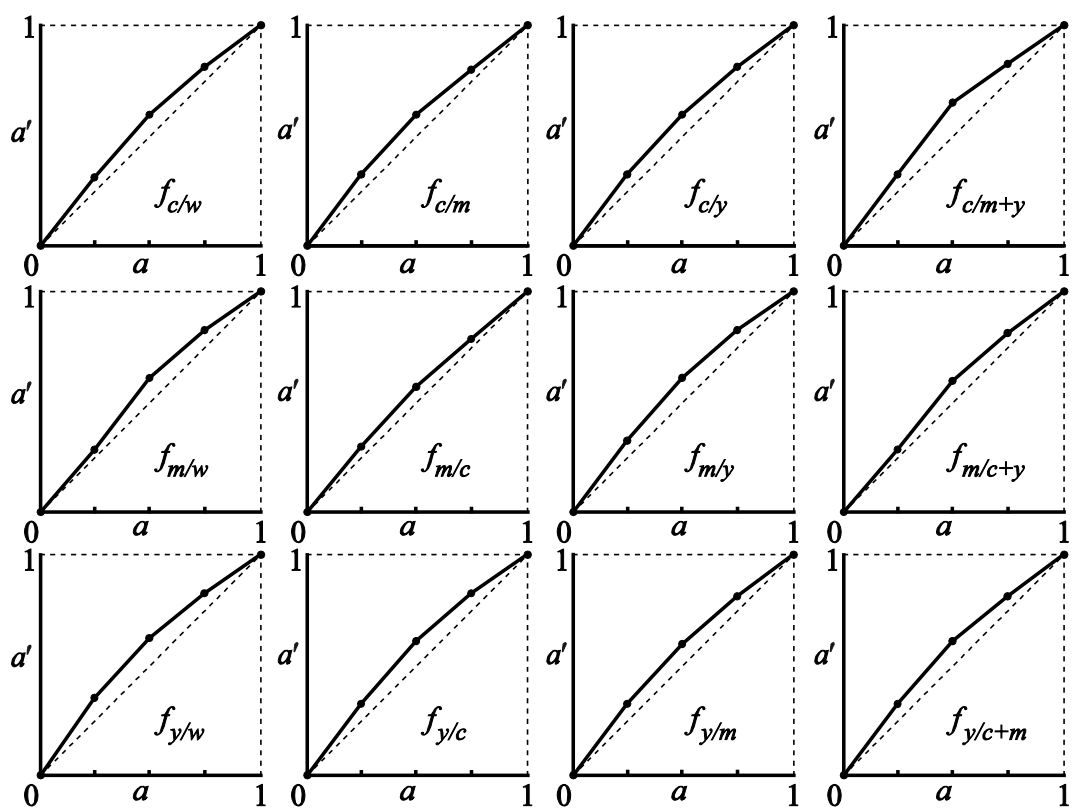

Figure 3. Example of ink spreading functions giving the effective surface coverage $a$ ' of each ink superposed with each colorant made of the other two inks at the surface coverages 1 or 0 , as functions of the nominal surface coverage $a$.
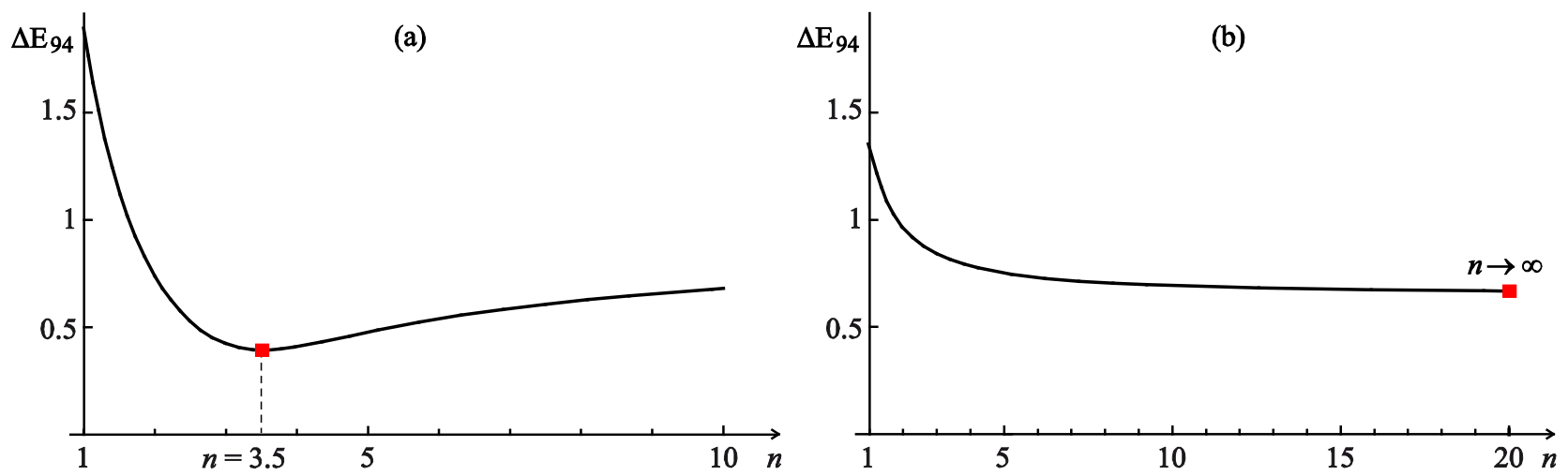

Figure 4. Variation as a function of the $n$ value of the average $\Delta \mathrm{E}_{94}$ computed from the predicted and measured spectral reflectances of the 36 single-ink halftones displayed in Figure 2, in the case of (a) inkjet CMY colors printed at 150 lpi on glossy coated paper and (b) in the case of CMY colors printed at 100 lpi on common office paper.

\subsection{Prediction}

Once the ink spreading functions have been obtained, the spectral reflectance of any halftone can be predicted. Let us consider a halftone where the cyan, magenta and yellow ink are printed at the nominal surface coverages $c_{0}, m_{0}$, and $y_{0}$. These surface coverages are converted into effective ink surface coverages $c, m$ and $y$ by a weighted average of the ink spreading functions in order to account for the superposition-dependent ink spreading [8]. The weights are expressed by the surface coverages of the respective colorants on which the ink halftone is superposed. For example, the weight of the ink spreading function $f_{c}$ (cyan halftone over white colorant) is $(1-m)(1-y)$. In the case of three halftoned inks, effective surface coverages are obtained by performing a few iterations with the following equations: 


$$
\begin{aligned}
& c=(1-m)(1-y) f_{c / w}\left(c_{0}\right)+m(1-y) f_{c / m}\left(c_{0}\right)+(1-m) y f_{c / y}\left(c_{0}\right)+m y f_{c / m+y}\left(c_{0}\right) \\
& m=(1-c)(1-y) f_{m / w}\left(m_{0}\right)+c(1-y) f_{m / c}\left(m_{0}\right)+(1-c) y f_{m / y}\left(m_{0}\right)+c y f_{m / c+y}\left(m_{0}\right) \\
& y=(1-c)(1-m) f_{y / w}\left(y_{0}\right)+c(1-m) f_{y / c}\left(y_{0}\right)+(1-c) m f_{y / m}\left(y_{0}\right)+c m f_{y / c+m}\left(y_{0}\right)
\end{aligned}
$$

For the first iteration, $c=c_{0}, m=m_{0}$ and $y=y_{0}$ are taken as initial values on the right side of the equations. The obtained values of $c, m$ and $y$ are then inserted again into the right side of the equations, which gives new values of $c, m$, $y$ and so on, until the values of $c, m, y$ stabilize. They are then plugged into Eq. (1) in order to obtain the effective surface coverages of the eight colorants, which are finally used in Eq. (3) to predict the spectral reflectance of the halftone.

\subsection{Spectral transmittance of halftone colors}

It has been recently shown that the spectral Yule-Nielsen transform applies to the spectral transmittance of halftone colors, either printed on paper [15] or on transparency films [16], in a similar way as for reflectances:

$$
T(\lambda)=\left[\sum_{k=1}^{8} a_{k} T_{k}^{1 / n}(\lambda)\right]^{n}
$$

where $T(\lambda)$ denotes the spectral transmittance of the halftone, $a_{i}$ the surface coverage in the halftone of each colorant $i$ whose spectral reflectances $T_{i}(\lambda)$ is measured using a spectrophotometer in transmittance mode, e.g. the X-Rite Color i7 instrument in total transmittance mode (diffuse: $0^{\circ}$ geometry).

Note that for a same halftone sample, the reflectance and transmittance equations may involve different $n$ values because scattering phenomena are different in reflection and transmission. The ink spreading functions may also be different and it is recommended to compute them from reflectance measurements for reflectance predictions, and from transmittance measurements for transmittance predictions.

\section{GRAPHICAL ANALYSIS OF THE YULE-NIELSEN TRANSFORM}

Although the Yule-Nielsen equation in known to be empirical and its physical justification has not been completely clarified despite several attempts [17, 18, 19], one generally observes good agreement between predicted and measured spectra for a wide range of printing techniques. However, it is rather difficult to interpret precisely the deviations that are sometimes observed between them. We thus propose a graphical method permitting the comparison in a more intelligible way in the case of single-ink halftones.

\subsection{Yule-Nielsen function and Fulltone-to-halftone reflectance diagram}

Let us denote as $R_{0}, R_{a}$ and $R_{1}$ the spectral reflectances of patches where the ink has the respective surface coverages of $0, a$ and 1 . The Yule-Nielsen equation for a single-ink halftone, already given in Eq. (4), is written:

$$
R_{a}(\lambda)=\left[\left(1-a^{\prime}\right) R_{0}^{1 / n}(\lambda)+a^{\prime} R_{1}^{1 / n}(\lambda)\right]^{n}
$$

where $a^{\prime}$ denotes the effective surface coverage of the ink corresponding to the nominal surface coverage $a$. Using the notations $y=R_{a} / R_{0}$ and $x=R_{1} / R_{0}$, Eq. (8) can also be written, for each wavelength:

$$
y=\left[1-a^{\prime}+a^{\prime} x^{1 / n}\right]^{n}
$$

We will call "Yule-Nielsen function" the function $x \rightarrow\left(1-a^{\prime}+a^{\prime} x^{1 / n}\right)^{n}$. By plotting it for given values of $n$ and a, one obtains a curve showing graphically the relationship between fulltone and halftone reflectances normalized by the unprinted support reflectance (Figure 5). When the ink is nonabsorbent at a given wavelength, we have $R_{a}=R_{1}=R_{0}$, therefore $x=y=1$. The Yule-Nielsen function thus satisfies $f(1)=1$ and the curve passes through the point $(1,1)$ in the diagram. When the ink is fully absorbing, we have $R_{a}=R_{1}=0$, therefore $x=0$ and $y=\left(1-a^{\prime}\right)^{1 / n}$.

In the special case where $n=1$, the Yule-Nielsen equation becomes the Neugebauer equation (2) and the Yule-Nielsen function is a straight line that intercepts the ordinate axis in $y=1-a^{\prime}$. It is tangent to the Yule-Nielsen function in $x=1$, i.e. the relationship between halftone and fulltone reflectance is almost linear when the fulltone has nearly the same reflectance as the unprinted support, therefore when the ink is almost not absorbing. 
SPIE Electronic Imaging - Color Imaging XIX: Displaying, Processing, Hardcopy, and Applications, 2-6 February 2014, 9015-27.
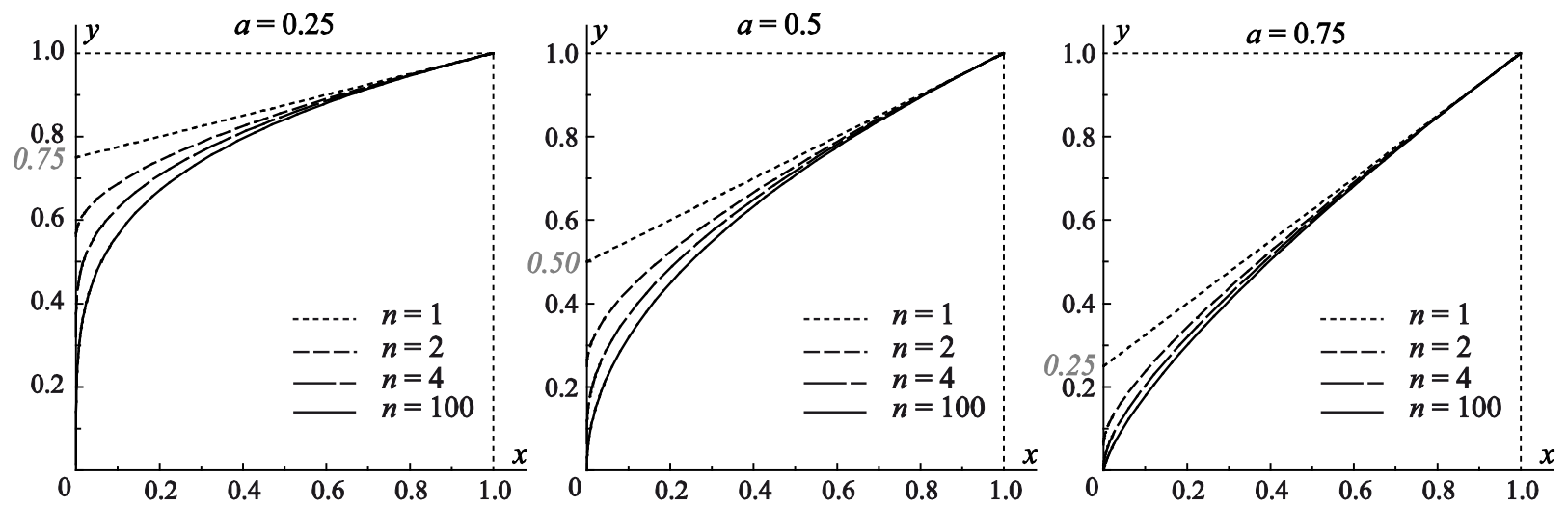

Figure 5. Plots of the Yule-Nielsen function defined by Eq. (9) for various $n$-values and three different surface coverages $a$.

This relationship can be verified experimentally from the measurements of $R_{0}(\lambda), R_{a}(\lambda)$ and $R_{1}(\lambda)$, which provides as many points as values contained in the measured spectra. We obtain a "fulltone-to-halftone diagram" as the ones showed in Figures 6, 7 and 8. The spectral reflectances needed to plot these diagrams are systematically measured for the calibration of the ink spreading functions.

Eq. (9) can also be written

$$
y^{1 / n}=1-a^{\prime}+a^{\prime} x^{1 / n},
$$

which means that the Yule-Nielsen model assumes a linear relationship between $\left(R_{a} / R_{0}\right)^{1 / n}$ and $\left(R_{1} / R_{0}\right)^{1 / n}$. This can also be experimentally verified in a "transformed halftone-to-fulltone diagram" (see Figures 6 and 7).

\subsection{Physical meaning of the Yule-Nielsen function}

The ratio $x=R_{1} / R_{0}$ can be interpreted as the effective transmittance of the ink layer for the average light path of light in the fulltone print. In a halftone, we may consider the layer as composed of absorbing areas with effective transmittance $x$ and non-absorbing areas with effective transmittance 1 . In absence of scattering, the effective transmittance of the halftone layer would be $y=1-a^{\prime}+a^{\prime} x$, which is the Neugebauer function. But since the layer is generally scattering, we can consider that after transmission through a sublayer of relative thickness $1 / n$, therefore of transmittance $t=1-a+a x^{1 / n}$, light is mixed before entering the next sublayer. Since the light crosses $n$ sublayers of relative thickness $1 / n$, the transmittance of the whole layer is $t^{n}$. This yields the Yule-Nielsen function defined by Eq. (9).

Although this interpretation is not realistic if one imagines light trajectories in the printing material, it has at least a physical meaning in term of statistical light transfers. A light scattering model would be necessary to establish the equivalence between this simple model and a more rigorous description of the propagation of light in the scattering halftone. But the experience shows that this equivalence must exist when the Yule-Nielsen model is capable of accurate prediction of colored halftones. Note that this interpretation of the Yule-Nielsen transform has been recently used to define a hybrid additive-subtractive color mixing system [20].

\subsection{Illustration of the graphical method with halftones printed in inkjet}

Figures 6 illustrates the graphical method through the example of a halftone printed with either cyan, magenta or yellow ink at nominal surface coverage 0.5 by using a periodical clustered dot halftoning at $150 \mathrm{lpi}$, and printed on Canon PP201 $260 \mathrm{~g} / \mathrm{m}^{2}$ glossy paper (same paper as for the experiment presented in Figure 4) with the Canon Pixma Pro9500 inkjet printer. The spectral reflectances of the unprinted paper, $R_{0}(\lambda)$, of the halftones, $R_{a}(\lambda)$, and of the fulltones, $R_{1}(\lambda)$, were measured from $380 \mathrm{~nm}$ to $730 \mathrm{~nm}$ in steps of $10 \mathrm{~nm}$ (36 wavebands) with the X-rite i1 spectrophotometer based on the $45^{\circ}: 0^{\circ}$ geometry. These spectra are plotted in the top row of the figure. In dashed line are plotted the spectral reflectances of the halftones predicted by the spectral Yule-Nielsen model with the optimal $n=3.5$ fitted in the calibration step (see. Section 2.3) and the effective surface coverage $a^{\prime}$ obtained from equation (5) and specified on top of each column. The fulltone-to-halftone diagrams, in the middle row, show the 36 points $\left(R_{1} / R_{0} ; R_{a} / R_{0}\right)$ computed from the measured spectral reflectances, as well as the Yule-Nielsen function given by Eq. (9) with $n=3.5$ (solid line) and the Neugebauer function given by Eq. (9) with $n=1$ (dashed line) for the specified effective surface coverage $a^{\prime}$. At the bottom of the figure are shown the transformed fulltone-to-halftone diagrams. 
SPIE Electronic Imaging - Color Imaging XIX: Displaying, Processing, Hardcopy, and Applications, 2-6 February 2014, 9015-27.

We see from the fulltone-to-halftone diagrams that the Yule-Nielsen function matches well the experimental points, which indicates that the Yule-Nielsen transform correctly models the nonlinear relationship between the reflectances of the halftone, the fulltone, and the unprinted paper. This is confirmed by the low $\Delta \mathrm{E}_{94}$ values computed from the measured and predicted spectra: 0.20 units for the cyan halftone, 0.55 units for the magenta halftone and 0.09 for the yellow halftone. This is also confirmed by the fact that the points $\left(x^{\prime} ; y^{\prime}\right)$, with $y^{\prime}=\left(R_{a} / R_{0}\right)^{1 / n}$ and $y^{\prime}=\left(R_{a} / R_{0}\right)^{1 / n}$, are well aligned and match the function $y^{\prime}=1-a^{\prime}+a^{\prime} x^{\prime}$ for the fitted values of $n$ and $a^{\prime}$.
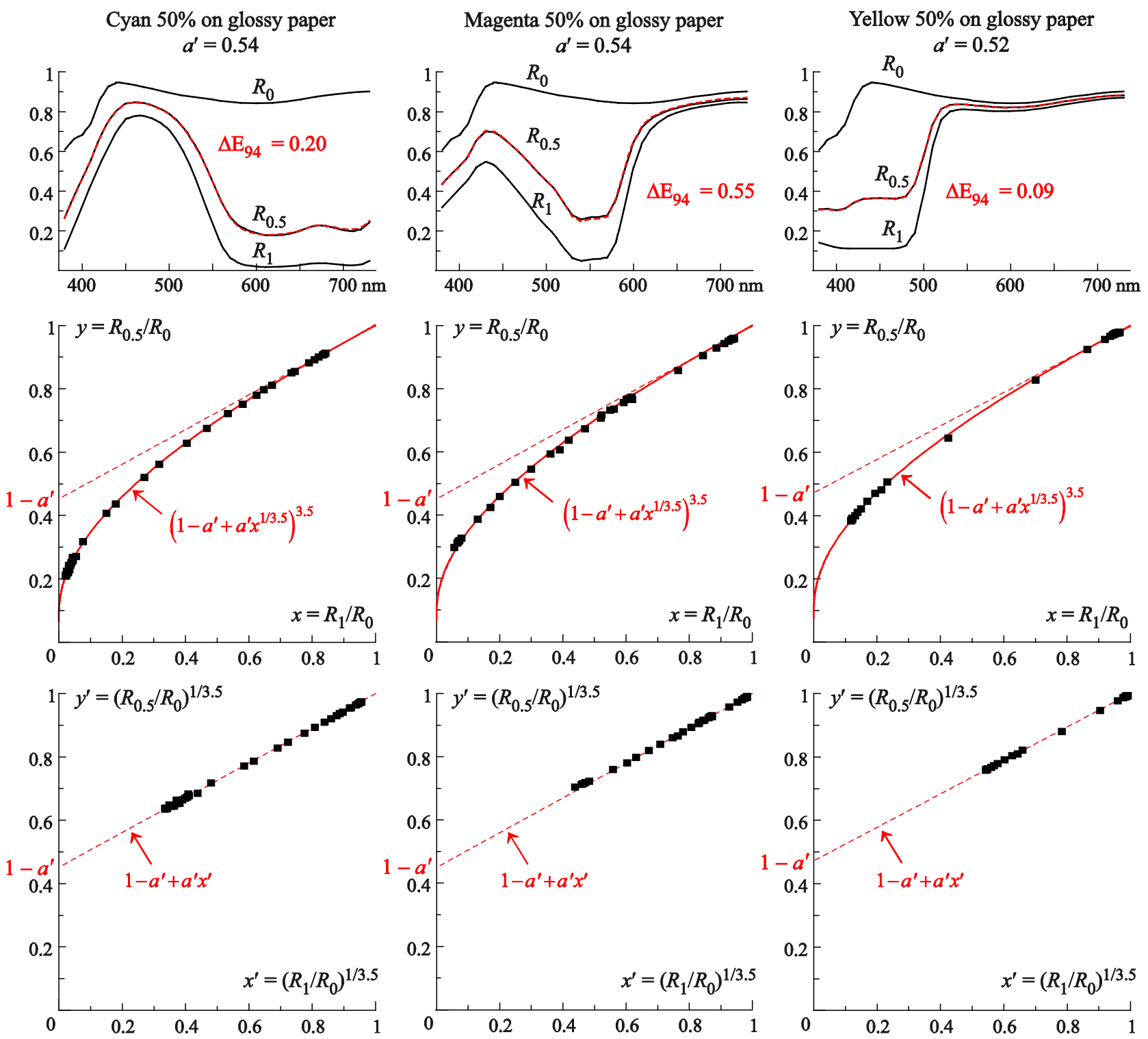

Figure 6. Top row: Measured spectral reflectances of cyan (left column), magenta (central column), and yellow (right column) halftones printed at the surface coverages 0.5 (reflectances $R_{0.5}$ ) and 1 (reflectances $R_{1}$ ) on glossy coated paper (reflectance $R_{0}$ ). Plots in dashed line represent the spectral reflectances of the halftones predicted by the spectral YuleNielsen model for the values of $a^{\prime}$ specified on top of each column. The CIELAB $\Delta \mathrm{E}_{94}$ values assess the deviation between predictions and measurements. Central row: Fulltone-to-halftone diagrams showing the points $\left(R_{1} / R_{0} ; R_{a} / R_{0}\right)$ deduced from the measured spectra in the different wavebands, as well as the Yule-Nielsen function given by Eq. (9) with $n=3.5$ (solid line) and the Neugebauer function, for the specified value of $a^{\prime}$. Bottom row: Transformed fulltone-to-halftone diagrams showing the experimental points $\left(\left(R_{1} / R_{0}\right)^{1 / n} ;\left(R_{a} / R_{0}\right)^{1 / n}\right)$ for $n=3.5$. 
SPIE Electronic Imaging - Color Imaging XIX: Displaying, Processing, Hardcopy, and Applications, 2-6 February 2014, 9015-27.

In order to verify the prediction accuracy of the model over a larger set of colors, we printed 125 halftones with the same printer, same inks, same paper and same halftoning technique as the colors used for Figure 6 and measured them using the same spectrophotometer. These 125 colors correspond to all the combinations of cyan, magenta and yellow inks printed at the nominal surface coverages $0,0.25,0.5,0.75$ and 1 . The average $\Delta \mathrm{E}_{94}$ value over these 125 colors is 0.51 units, and the $95 \%$-quantile of the $125 \Delta \mathrm{E}_{94}$ values is 1.12 units. We can therefore conclude that the Yule-Nielsen model is well performing for this type of print.

\subsection{Limitations of the Yule-Nielsen function}

Let us now study halftones printed with the same printer, the same inks and the same halftoning technique on a nonfluorescent, non-calendered and noticeably porous $80 \mathrm{~g} / \mathrm{m}^{2}$ paper called Biotop. Figure 7 presents similar graphs as those presented in Figure 6, corresponding to the cyan ink halftones at $0.25,0.5$ and 0.75 nominal surface coverages. The Yule-Nielsen functions where calculated with an optimal $n$ value of 100 .
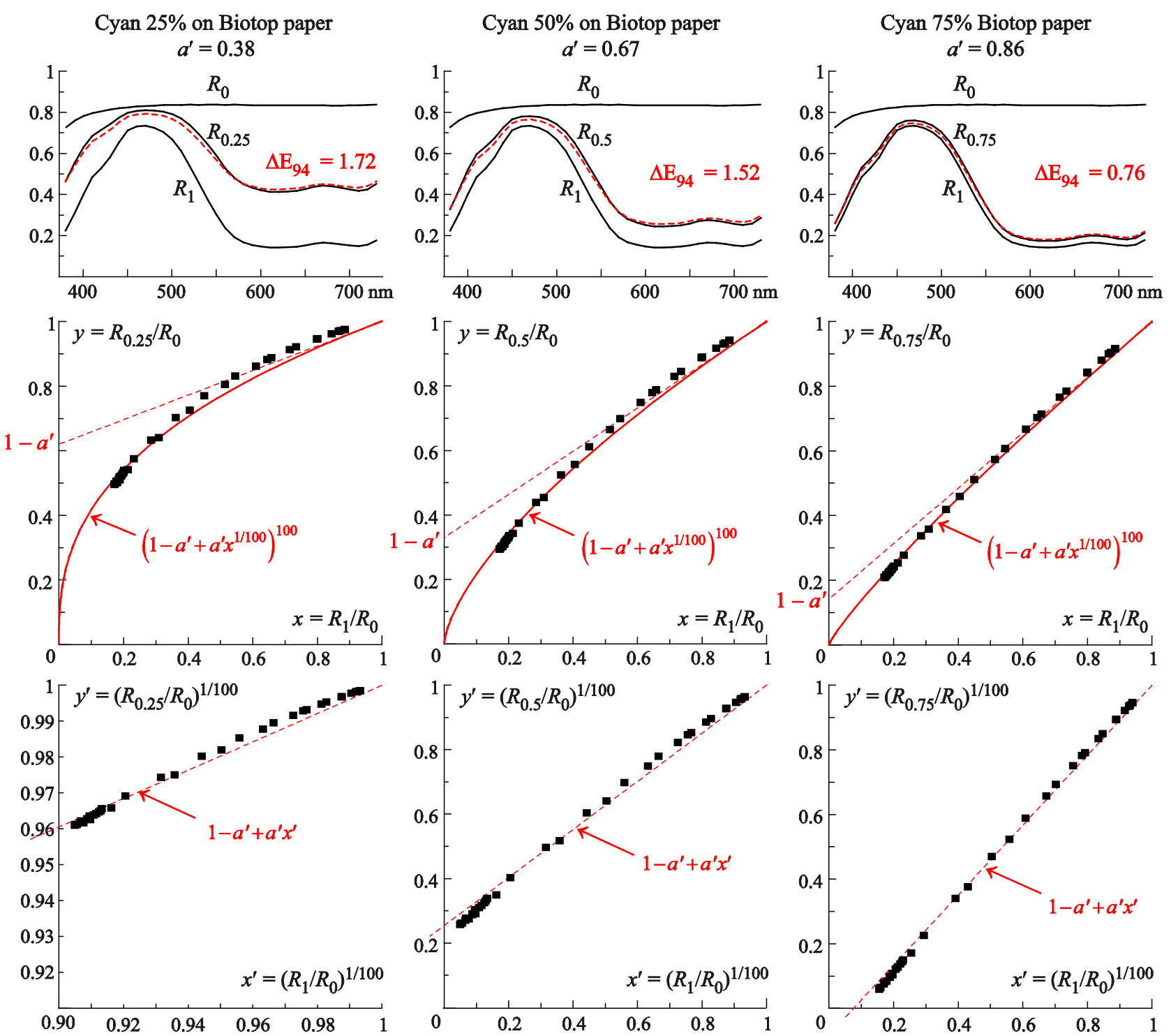

Figure 7. Similar graphs as in Figure 6 for cyan ink halftones printed at $0.25,0.5$ and 0.75 nominal surface coverages on Biotop non-calendered paper. The Yule-Nielsen functions were calculated with $n=100$. 
SPIE Electronic Imaging - Color Imaging XIX: Displaying, Processing, Hardcopy, and Applications, 2-6 February 2014, 9015-27.

This example shows that good agreement between prediction and measurement cannot be achieved for all types of prints: Despite the optimized $n$ and $a$ ' values, the Yule-Nielsen functions do not match the experimental points satisfyingly, and the points $\left(x^{\prime} ; y^{\prime}\right)$, with $x^{\prime}=\left(R_{1} / R_{0}\right)^{1 / n}$ and $y^{\prime}=\left(R_{a} / R_{0}\right)^{1 / n}$, are not aligned. Consequently, the prediction accuracy of the spectral Yule-Nielsen model is poorer: the $\Delta \mathrm{E}_{94}$ values for the halftones printed at 0.25 and 0.5 surface coverage are beyond the value of the just noticeable color difference threshold $\left(\Delta \mathrm{E}_{94}=1\right)$. On a set of 64 patches designed for verification (all combinations of the cyan, magenta and yellow inks printed at the nominal surface coverages $0.13,0.38$, 0.63 and 0.88 ), we obtained an average $\Delta \mathrm{E}_{94}$ value of 1.23 units, and a $95 \%$-quantile of 2.36 units.
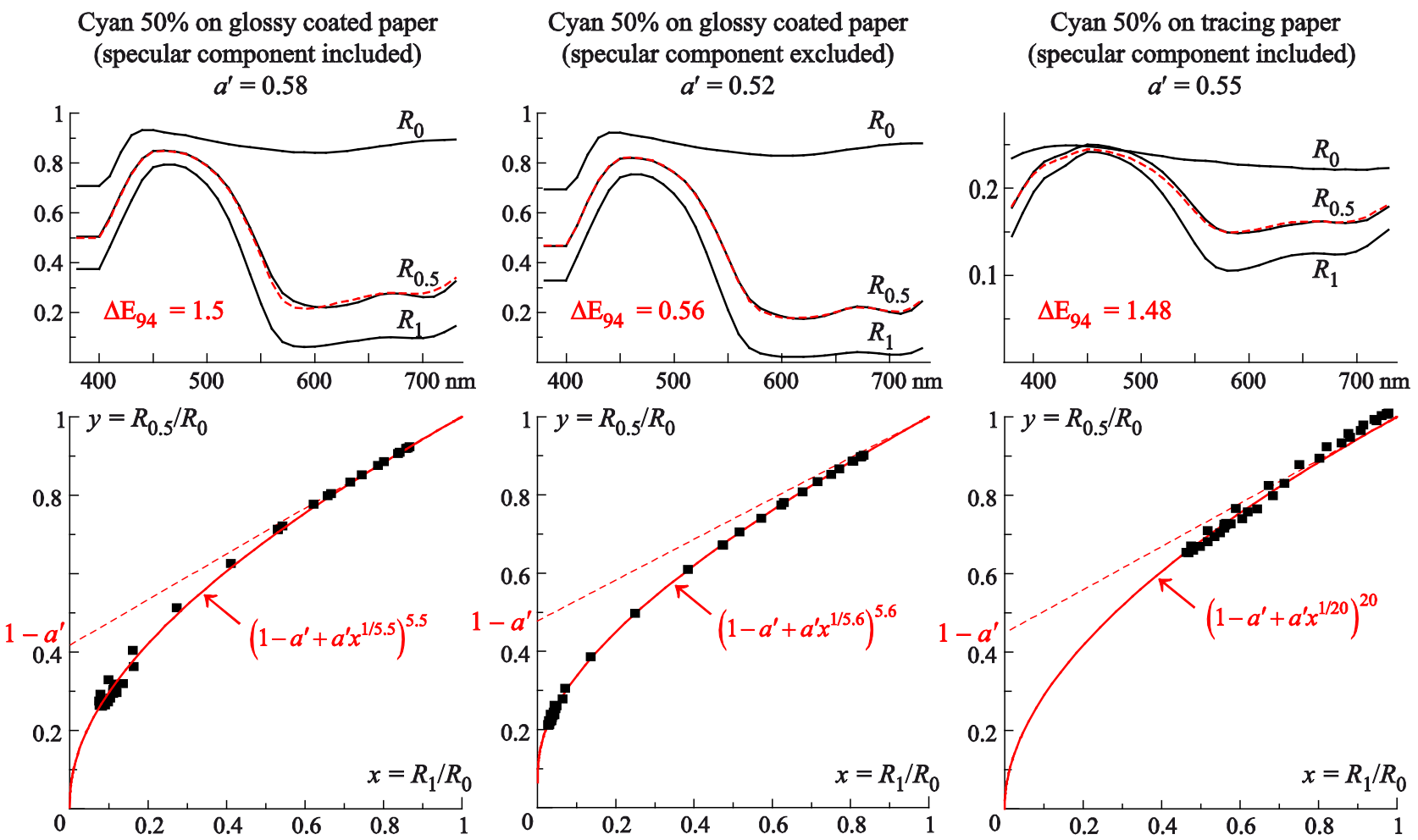

Figure 8. Spectral reflectances and fulltone-to-halftone diagrams for cyan ink halftones printed at 0.5 nominal surface coverage on the PP201 glossy coated paper (left and center) and on tracing paper (right). The spectral measurements are based on the diffuse: $8^{\circ}$ geometry with specular component included (left and right) and excluded (center).

Figure 8 shows three other examples of cyan ink halftone printed at nominal surface coverage 0.5 . On the left and the center of the figure, the studied sample is the same as in the left column of Figure 6 but instead of measuring its spectral reflectance with the X-rite il spectrophotometer based on the $45^{\circ}: 0^{\circ}$ geometry, we used the X-rite Color i7 spectrophotometer based on the diffuse: $8^{\circ}$ geometry in either specular reflection included mode (left column) or specular reflection excluded mode (central column). The difference that we observe between the two measurement modes is due to the fact that the cyan ink displays a reddish sheen in the specular direction which is more pronounced in the fulltone than in halftones. This sheen is responsible for the deviation between measured and predicted spectra beyond $570 \mathrm{~nm}$ in the specular reflection included mode. In the fulltone-to-halftone diagram, the left-most experimental points in the graph follow "u"-shaped line and are thus located on either side of the Yule-Nielsen function, which means that $y$ have different values for a same value of $x$. No function can reproduce such an incurved line since $y$ can have only one value for one $x$ value. However, the global prediction accuracy over the 125 halftone colors described in the previous section is still acceptable because the colored sheen is visible in a limited number of halftones, i.e. those where the cyan ink has a high surface coverage and is not superposed with other inks. The average $\Delta \mathrm{E}_{94}$ value obtained with the optimal $n=5.5$ was 0.91 units, therefore below the just noticeable color difference, and the $95 \%$-quantile 1.95 units. The prediction accuracy is nevertheless poorer than in the specular reflection excluded mode where the reddish sheen is not observed: for the same set of 125 halftone colors, the average $\Delta \mathrm{E}_{94}$ value obtained with the optimal $n=5.6$ was 0.62 units and the $95 \%$-quantile 1.19 units, therefore comparable with the prediction accuracy achieved with measurements based on the $45^{\circ}: 0^{\circ}$ geometry. 
SPIE Electronic Imaging - Color Imaging XIX: Displaying, Processing, Hardcopy, and Applications, 2-6 February 2014, 9015-27.

The graphs on the right of Figure 8 show the case of the same cyan ink printed at 120 lpi on Canson $90 \mathrm{~g} / \mathrm{m}^{2}$ tracing paper. Since the tracing paper is semi-transparent, the reflectance is much lower in comparison to the other papers (see the ordinate scale of the plotted reflectances). For this sample, the experimental points are dispersed on either side of the Yule-Nielsen function. The prediction accuracy achieved on a set of 40 CMY halftones is very poor: we obtained an average $\Delta \mathrm{E}_{94}$ value of 2.99 units and a $95 \%$-quantile of 4.58 units.

\section{ATTEMPTS TO IMPROVE THE YULE-NIELSEN MODEL}

Rewriting the Yule-Nielsen equation (3) as

$$
R(\lambda)=f^{-1}\left[\sum_{i=1}^{8} a_{i} f\left[R_{i}(\lambda)\right]\right]
$$

with the function $f: x \rightarrow x^{1 / n}$, and the reverse function $f^{-1}: y \rightarrow y^{1 / n}$ can be a source of inspiration to find other functions $f$ in order to improve the spectral Yule-Nielsen model when it fails to provide accurate prediction accuracy. The proposition by Lewandowski et al. [19] to select a negative $n$ value in the Yule-Nielsen model, while $n$ is rather supposed higher than 1, can be considered as a first example of extended Yule-Nielsen function.

The new functions $f_{i}$ should be defined and continuous on $[0,1]$ and satisfy the condition $f_{i}(1)=1$. The reflectance $R_{a}$ of a single-ink halftone of surface coverage $a^{\prime}$ is written:

$$
R_{a}=f_{i}^{-1}\left(\left(1-a^{\prime}\right) f_{i}\left(R_{1}\right)+a^{\prime} f_{i}\left(R_{1}\right)\right)
$$

where $R_{0}$ and $R_{1}$ denote the reflectances of the printing support without, respectively with ink. It can also be written

$$
\frac{f_{i}\left(R_{a}\right)}{f_{i}\left(R_{0}\right)}=1-a^{\prime}+a^{\prime} \frac{f_{i}\left(R_{1}\right)}{f_{i}\left(R_{0}\right)}
$$

but not

$$
f_{i}\left(R_{a} / R_{0}\right)=1-a^{\prime}+a^{\prime} f_{i}\left(R_{1} / R_{0}\right)
$$

except for the functions of the form $f: x \rightarrow x^{\alpha}$ where $\alpha$ is a real number (i.e. those on which is based the classical YuleNielsen model). The fulltone-to-halftone diagram, based on the values $R_{1} / R_{0}$ and $R_{a} / R_{0}$, is therefore not helpful to optimize the new function, but we can check graphically in the transformed fulltone-to-halftone diagram whether, for the selected function, the points $\left(f_{i}\left(R_{a}\right) / f_{i}\left(R_{0}\right) ; f_{i}\left(R_{1}\right) / f_{i}\left(R_{0}\right)\right)$ are aligned.

The searching of alternative functions is empirical and may be tedious. A first idea to generate new functions is to make simple transforms on the classical function itself, by introducing for example a shift:

$$
f_{1}: x \rightarrow(x-s)^{1 / n} ; \quad f_{1}^{-1}: y \rightarrow y^{n}+s
$$

where $s$ is a real number that somehow represents surface reflection, not subject to optical dot gain. Note that since this function does not satisfy the condition $f(1)=1$, the $s$ value should be rather small.

The classical function $f$ can also be modified in the following way:

$$
f_{2}: x \rightarrow\left(q x-(1-q) x^{2}\right)^{1 / n} ; \quad f_{2}^{-1}: y \rightarrow \frac{\left[q-\sqrt{q^{2}+4(1-q) y^{n}}\right]}{2(q-1)}
$$

and function $f_{1}$ and $f_{2}$ can be combined:

$$
f_{3}: x \rightarrow\left(q x-(1-q)(x-s)^{2}\right)^{1 / n} ; \quad f_{3}^{-1}: y \rightarrow \frac{q-\sqrt{q^{2}+4(1-q) y^{n}}}{2(q-1)}+s
$$

Another transform of the function $f_{1}$ is 
SPIE Electronic Imaging - Color Imaging XIX: Displaying, Processing, Hardcopy, and Applications, 2-6 February 2014, 9015-27.

$$
f_{4}: x \rightarrow \ln \left[(x-s)^{1 / n}-1+b\right] / \ln (b) ; \quad f_{4}^{-1}: y \rightarrow\left(b^{x}+1-b\right)^{n}+s
$$

where "In" denotes the natural logarithm function. More complicated functions can be found, for example

$$
f_{5}: x \rightarrow \frac{1}{\omega} \operatorname{arcsh}(n \ln (x \oplus 1 \dashv s)) ; 1 f_{5}^{-1}: y \rightarrow \exp (\sinh (y) n)-+s
$$

where "arcsh" denotes the inverse of the hyperbolic sine function, and $\omega=\operatorname{arcsh}(n \ln 2)$.

These new functions $f_{1}$ to $f_{5}$ were tested on the 64 halftones printed on Biotop paper already presented in Section 3.2 and Figure 7. For the model calibration and the predictions, similar procedure is followed as the one detailed in Section 2 by simply replacing the classical function $f$ with the considered function $f_{i}$. The prediction accuracy yielded by each function for the 64 halftones is presented in Table 1, as well as the values of the tunable parameters which were fitted in order to obtain the lowest average $\Delta \mathrm{E}_{94}$ value. The five functions yield very similar accuracy, which is slightly better than with the classical function $f$ although the average $\Delta \mathrm{E}_{94}$ value remains beyond the just noticeable color difference $\left(\Delta \mathrm{E}_{94}=1\right)$. The improvement, modest but appreciable, is partly explained by the introduction of additional tunable parameters, but also by the fact that the experimental points are better aligned in the transformed fulltone-to-halftone diagram, as shown in Figure 9 through the example of the cyan halftone printed at the surface coverage 0.5 on the Biotop paper (same sample as the one studied in the central column of Figure 7).

Table 1. Performance of the spectral Yule-Nielsen model based on the classical function $f$ and on transformed functions $f_{i}$ on 64 halftones covering the printer's gamut printed on Biotop non-calendered paper.

\begin{tabular}{|llcc|}
\hline Function & Parameters & Average $\Delta \mathrm{E}_{94}$ & 95-quantile $\Delta \mathrm{E}_{94}$ \\
\hline$f: x \rightarrow x^{1 / n}$ & $n=100$ & 1.23 & 2.36 \\
$f_{1}: x \rightarrow(x-s)^{1 / n}$ & $n=20 ; s=0.09$ & 1.12 & 2.10 \\
$f_{2}: x \rightarrow\left(q x-(1-q) x^{2}\right)^{1 / n}$ & $n=100 ; q=0.12$ & 1.11 & 2.12 \\
$f_{3}: x \rightarrow\left(q x-(1-q)(x-s)^{2}\right)^{1 / n}$ & $n=50 ; s=0.06 ; q=-0.05$ & 1.12 & 2.09 \\
$f_{4}: x \rightarrow \ln \left[(x-s)^{1 / n}-1+b\right] / \ln (b)$ & $n=2 ; b=1.7 ; s=0.12$ & 1.11 & 2.16 \\
$f_{5}: x \rightarrow \operatorname{arcst}(n \ln (x+1-s)) /$ & $n=100 ; s=0.09$ & 1.14 & 2.24 \\
\hline
\end{tabular}

Classical function $f$ with $a^{\prime}=0.67$

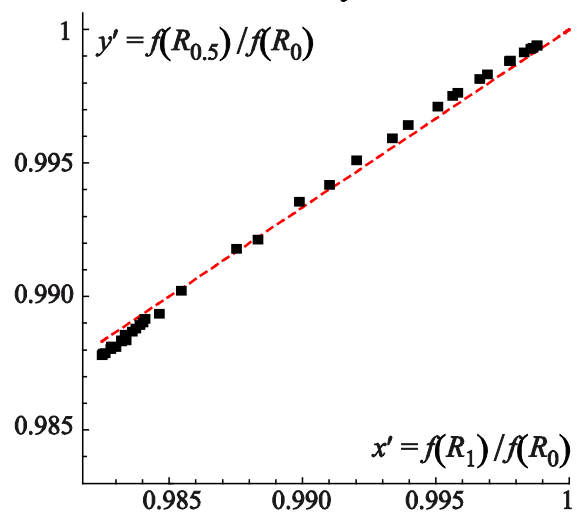

Function $f_{1}$ with $a^{\prime}=0.61$

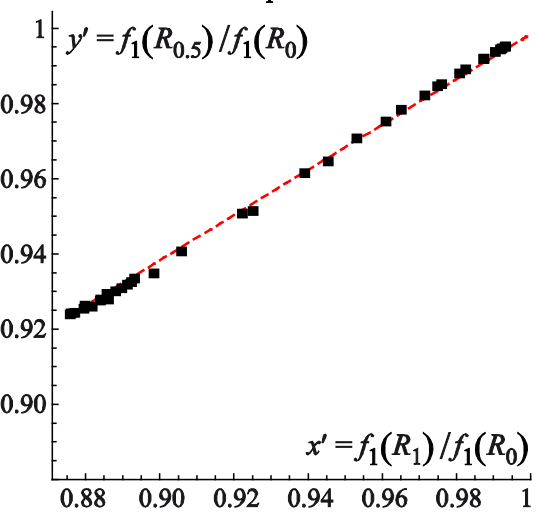

Function $f_{2}$ with $a^{\prime}=0.61$

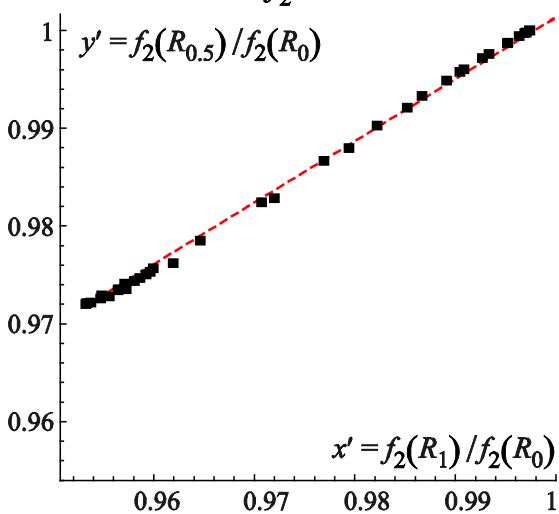

Figure 9. Transformed fulltone-to-halftone diagrams representing the points $\left(f_{i}\left(R_{1}\right) / f_{i}\left(R_{0}\right) ; f_{i}\left(R_{0.5}\right) / f_{i}\left(R_{0}\right)\right)$ issued from the measured spectral reflectances of the Biotop paper with no ink (reflectance $R_{0}$ ), with full coverage ink (reflectances $R_{1}$ ) and with halftoned ink at 0.5 nominal surface coverage (reflectances $R_{0.5}$ ), for the classical function $f$ and the functions $f_{1}$ and $f_{2}$ computed with the parameters presented in Table 1 . The lines of equation: $y^{\prime}=1-a^{\prime}+a^{\prime} x^{\prime}$, where $a^{\prime}$ is the effective surface coverage of the halftone fitted for each function, are plotted in dashed line. 
SPIE Electronic Imaging - Color Imaging XIX: Displaying, Processing, Hardcopy, and Applications, 2-6 February 2014, 9015-27.

The three diagrams in Figure 9 have been generated by the classical function $f$ and the functions $f_{1}$ and $f_{2}$ (those obtained with the functions $f_{3}$ to $f_{5}$, quasi-identical to these latters, are not reproduced). The effective surface coverages $a^{\prime}$ specified on top of the graphs were fitted for each function. In this example, functions $f_{1}$ and $f_{2}$ yield the same $a^{\prime}$-value, lower than the one fitted with $f$. This indicates that $f_{1}$ and $f_{2}$ model stronger optical dot gain than $f$. We also see that the experimental points are closer to the straight line of equation: $y^{\prime}=1-a^{\prime}+a^{\prime} x^{\prime}$ with the new functions than for the classical one. Is seems that the improvement of accuracy is due to the fact that the line passes through the points corresponding to the highest absorbance of the ink (left-most points in the diagram), which is due to the fact that the $\Delta \mathrm{E}_{94}$ value is more sensible to spectral deviations in wavelengths domains where the spectrum is low.

\section{CONCLUSIONS}

Despite its physical base is not completely established yet, the spectral Yule-Nielsen model improved by Hersch and Crété's ink spreading assessment method provides a high prediction accuracy for most types of halftone prints. However, it is less performing for certain types of printed surfaces, especially when the printing support is lowly reflective like tracing paper, or when the surface of the print has a colored sheen in the specular direction. The graphic method proposed in this paper enables verifying the relevance of the Yule-Nielsen transform for the considered printing setup, by plotting for single-ink halftones the Yule-Nielsen function $y(x)=\left(1-a^{\prime}+a^{\prime} x^{1 / n}\right)^{n}$ with the optimal $n$ and $a^{\prime}$ values computed in the calibration step and comparing it with the points $\left(R_{1} / R_{0} ; R_{a} / R_{0}\right)$ computed in the different wavebands from the measured spectral reflectances of the paper, $R_{0}(\lambda)$, of the halftone, $R_{a}(\lambda)$, and of the fulltone of the corresponding ink, $R_{1}(\lambda)$. As an alternative, one can check whether the points $\left(\left(R_{1} / R_{0}\right)^{1 / n} ;\left(R_{a} / R_{0}\right)^{1 / n}\right)$ are aligned and compare them to the function $y\left(x^{1 / n}\right)=1-a^{\prime}+a^{\prime} x^{1 / n}$.

It may happen that the $\left(R_{1} / R_{0} ; R_{a} / R_{0}\right)$ points do not draw a well defined curve in the fulltone-to-halftone diagram, i.e., that at wavelength where the unprinted paper and the fulltone have respectively same reflectance the halftone has different reflectances, due for example to optical phenomena such as fluorescence, too strong absorption or color sheen at the surface. Except these optical phenomena are explicitly modeled, the spectral Yule-Nielsen model is incapable of predicting these differences of reflectance and the prediction accuracy in this case is generally poorer.

In the case where the spectral Yule-Nielsen model is judged to be not accurate enough but the $\left(R_{1} / R_{0} ; R_{a} / R_{0}\right)$ points draw a well defined line, one can try to find another function than the $x^{1 / n}$ function in order to improve the prediction accuracy. A few ones have been proposed in this paper, but other ones can be invented. The improvement of prediction accuracy may be modest, as in the examples presented here, but still appreciable.

\section{ACKNOWLEDGEMENT}

The author would like to thank Baptiste Paul, Serge Mazauric and Jacques Machizaud for their contribution to this study.

\section{REFERENCES}

[1] Hersch, R.D. and Hébert, M., "Interaction between light, paper and color halftones: challenges and modelization approaches," Proc. IS\&T CGIV, 1-7 (2006).

[2] Kipphan, H. [Handbook of Print Media] Springer Ed., 555-562, (2001).

[3] Viggiano, J. A. S., "The Color of Halftone Tints," Proc. TAGA, 647-661 (1985).

[4] Balasubramanian, R., "Optimization of the spectral Neugebauer model for printer characterization," J. Electronic Imaging 8(2), 156-166 (1999).

[5] Iino, K. and Berns, R.S., "Building color management modules using linear optimization I. Desktop,” J. Im. Sci. Technol. 42(1), 79-94 (1998).

[6] Iino, K. and Berns, R.S., "Building color management modules using linear optimization II. Prepress system for offset printing," Journal of Imaging Science and Technology, 42(2), 99-114 (1998).

[7] Xia, M., Saber, E., Sharma, G. and Tekalp, M., "End to end color printer calibration by total least squares regression," IEEE Trans. Image Processing, 8(5), 700-716 (1999).

[8] Hersch, R.D. and Crété, F., "Improving the Yule-Nielsen modified spectral Neugebauer model by dot surface coverages depending on the ink superposition conditions," SPIE Proc. 5667:434-445 (2005). 
SPIE Electronic Imaging - Color Imaging XIX: Displaying, Processing, Hardcopy, and Applications, 2-6 February 2014, 9015-27.

[9] Yule, J.A.C. and Nielsen, W.J., "The penetration of light into paper and its effect on halftone reproduction," Proc. TAGA 3, 65-76 (1951).

[10] Arney, J. S., "A Probability Description of the Yule-Nielsen Effect I," J. Im. Sci. Technol. 41(6), 633-636 (1997).

[11] Hébert, M. and Hersch, R. D., "Analyzing halftone dot blurring by extended spectral prediction models," J. Opt. Soc. Am. A 27, 6-12 (2010)

[12] Demichel, M. E., Procédés 26, 17-21 (1924).

[13] Neugebauer, H. E. J., "Die theoretischen Grundlagen des Mehrfarbendrucks," Zeitschrift fuer wissenschaftliche Photographie 36, 36-73, (1937), translated into English: The theoretical basis of multicolour letterpress printing, Color Res. App. 30, 322-331 (2005).

[14] Wyszecki, G. and Stiles, W. S., [Color Science: Concepts and Methods, Quantitative Data and Formulae, 2nd ed.], Wiley (1982).

[15] Hébert, M. and Hersch, R. D., "Yule-Nielsen based recto-verso color halftone transmittance prediction model," Applied Optics 50, 519-525 (2011).

[16] Machizaud, J. and Hébert, M., "Spectral reflectance and transmittance prediction model for stacked transparency and paper both printed with halftone colors," J. Opt. Soc. Am. A 29(8), 1537-1548 (2012).

[17] Ruckdeschel, F. R. and Hauser, O. G., "Yule-Nielsen effect in printing: a physical analysis," Applied Optics 17, 3376-3383 (1978)

[18] Arney, J. S. and Yamaguchi, S., "The Physics Behind the Yule-Nielsen Equation," IS\&T’s PICS Conference, 381-385 (1999).

[19] Lewandowski, A., Ludl, M., Byrne, G. and Dorffner, G., "Applying the Yule-Nielsen equation with negative n," J. Opt. Soc. Am. A, 23(8), 1827-1834 (2006).

[20] Simonot, L., and Hébert, M., "Between additive and subtractive color mixings: intermediate mixing models," J. Opt. Soc. Am. A, doc. ID 194145 (posted 13 November 2013, in press). 